\title{
Generalized Elastic Model: Fractional Langevin Description, Fluctuation Relation and Linear Response
}

\author{
A. Taloni ${ }^{1}$, A. Chechkin ${ }^{2,3}$, J. Klafter ${ }^{4}$ * \\ ${ }^{1}$ CNR-IENI, Via R. Cozzi 53, 20125 Milano, Italy \\ 2 Max-Planck-Institute for Physics of Complex Systems, Noethnitzer Str. 38 \\ D-91187 Dresden, Germany \\ 3 Akhiezer Institute for Theoretical Physics, NSC KIPT, Kharkov 61108, Ukraine \\ ${ }^{4}$ School of Chemistry, Tel Aviv University, Tel Aviv 69978, Israel
}

\begin{abstract}
The Generalized Elastic Model is a linear stochastic model which accounts for the behaviour of many physical systems in nature, ranging from polymeric chains to single-file systems. If an external perturbation is exerted only on a single point $\boldsymbol{x}^{\star}$ (tagged probe), it propagates throughout the entire system. Within the fractional Langevin equation framework, we study the effect of such a perturbation, in cases of a constant force applied. We report most of the results arising from our previous analysis and, in the present work, we show that the Fox $H$-functions formalism provides a compact, elegant and useful tool for the study of the scaling properties of any observable. In particular we show how the generalized Kubo fluctuation relations can be expressed in terms of $H$-functions.
\end{abstract}

Keywords and phrases: fractional Langevin equation, subdiffusion, Fox $H$-function, linear response

Mathematics Subject Classification: 82C31, 82C70, 60G22，33E20

\section{Introduction}

The generalized elastic model (GEM) has been firstly introduced in [1] through the following equation:

$$
\frac{\partial}{\partial t} \mathbf{h}(\boldsymbol{x}, t)=\int d^{d} x^{\prime} \Lambda\left(\boldsymbol{x}-\boldsymbol{x}^{\prime}\right) \frac{\partial^{z}}{\partial\left|\boldsymbol{x}^{\prime}\right|^{z}} \mathbf{h}\left(\boldsymbol{x}^{\prime}, t\right)+\boldsymbol{\eta}(\boldsymbol{x}, t),
$$

The general formulation in (1.1) is given for the $D$-dimensional stochastic field $\mathbf{h}$ defined in the $d$ dimensional infinite space $\boldsymbol{x}$. The white noise $\eta$ satisfies the fluctuation-dissipation (FD) relation, i.e.

$$
\left\langle\eta_{j}(\boldsymbol{x}, t) \eta_{k}\left(\boldsymbol{x}^{\prime}, t^{\prime}\right)\right\rangle=2 k_{B} T \Lambda\left(\boldsymbol{x}-\boldsymbol{x}^{\prime}\right) \delta_{j k} \delta\left(t-t^{\prime}\right)
$$

${ }^{*}$ Corresponding author. E-mail: alessandro.taloni@gmail.com 
$(j, k \in[1, D])$, where $\Lambda(\boldsymbol{r})=1 /|\boldsymbol{r}|^{\alpha}$ corresponds to the hydrodynamic friction kernel whose Fourier transform is

$$
\Lambda(\boldsymbol{q})=\frac{(4 \pi)^{d / 2}}{2^{\alpha}} \frac{\Gamma((d-\alpha) / 2)}{\Gamma(\alpha / 2)}|\boldsymbol{q}|^{\alpha-d}=A|\boldsymbol{q}|^{\alpha-d}
$$

if $\frac{d-1}{2}<\alpha<d$. The fractional derivative $\partial^{z} / \partial|\boldsymbol{x}|^{z}$, defined via its Fourier transform by [2]

$$
\mathcal{F}_{\boldsymbol{q}}\left\{\frac{\partial^{z}}{\partial|\boldsymbol{x}|^{z}}\right\} \equiv-|\boldsymbol{q}|^{z}
$$

has another common definition given in term of the Laplacian $\Delta$ as $\frac{\partial^{z}}{\partial|\boldsymbol{x}|^{z}}:=-(-\Delta)^{z / 2}[3]$. The GEM (1.1) accounts for the dynamics of polymers [4-6], elastic chains [7-9], membranes [5,10-13] and rough surfaces [14-17], among others. It also reproduces the anomalous diffusive behavior of systems such as crack propagation [18] and contact line of a liquid meniscus [19]. Each one of the above-mentioned physical systems corresponds to a given set of the parameters defining the GEM (1.1), namely $z, \alpha$ and $d$, with $\alpha=d$ in the case of $\Lambda(\boldsymbol{r})=\delta(|\boldsymbol{r}|)(A=$ const in (1.3)).

In Ref. [1] we derived a Langevin equation for the probe at a generic position $\boldsymbol{x}$ starting from the system Eq.(1.1): this equation turned out to be a fractional Langevin equation (FLE), i.e. an usual overdamped Langevin equation with the normal time derivative replaced by a fractional one. Generally speaking, in the last decade the number of papers devoted to FLE and its connection to anomalous diffusing systems has notably increased. In [20] FLE was used to furnish the adequate representation of the Brownian motion of a massive particle moving in a surrounding fluid, whose hydrodynamical effects come into play through the Basset-Boussinesq retarding force. Furthermore, in [21] FLE was connected to the fractional Brownian motion (FBM) [22]. So far, the most important application of FLE is in protein dynamics. Indeed FLE has been introduced to capture the equilibrium conformational fluctuations of a protein molecule [23]. The dynamics of the distance between the donor $(D)$ and the acceptor $(A)$ coordinates within a protein was shown to be reproduced, with an excellent degree of accuracy, by a FLE with an applied hookean force. The FLE has been also the subject of theoretical works, which investigated the correlation functions behaviours in presence of an external harmonic force [24,25], and in the absence of any [26]. See also the recent review [27] for more references and details.

Within the framework offered by GEM (1.1), FLE formalism has been successfully applied to the statistical field theory. The stochastic motion of a tagged point (hereafter named probe or tracer without distinction) on a membrane surface by instance, was shown to be represented by a FLE [11], in the situation on which such a probe was subject to a linear force mimicking the action of an optical or magnetic tweezer. The tracer height $h(\boldsymbol{x}, t)$ is a stochastic field indeed: it is a function of the time $t$ and of the bidimensional membrane parametrization coordinate $\boldsymbol{x}$. In single file systems, where a tagged Brownian particle (the probe) undergoes subdiffusive motion on the score of the hard-core collisions with the other identical one dimensional file particles, the FLE was first phenomenologically proposed [28] and afterwards rigorously derived to be the effective tracer stochastic equation [29]. Here the probe position is represented by $h(x, t)$ where $x$ stands for the particle ordering number along the file. Furthermore, the FLE description of a tagged monomer dynamics in polymeric chains has been recently introduced in [30]: in this case $\mathbf{h}(x, t)$ represents the 3 dimensional position of the tagged monomer (probe), and $x$ its position along the polymer backbone (curvilinear abscissa).

In [31] we furnished the analytic expression of the field correlation functions using the Fox $H$-function formalism [32-34]. We highlighted the valuable property that physical observables, such as, e.g., mean squared displacement or the structure factor, could have a compact and elegant expression in terms of the Fox $H$-functions. These functions gain more and more popularity among the scientific community, for their very general nature, which allows to tackle different phenomena in a unified and elegant framework. Applications include non-Debye relaxation processes [35], anomalous diffusion [36-38], reaction-diffusion equations [39], relaxation and reaction processes in disordered systems [40], fractional Schroedinger 
equation [41], to name a few. The book [42] serves a deep analysis of properties of the Fox $H$ functions. Recent monograph [43] lists many useful properties of the Fox $H$-functions, together with some applications, e.g., in astrophysics. The handbook [44] contains the list of useful properties and integrals of the Fox $H$-functions.

In a recent publication [45] we added a localized external force to the system (1.1), namely a force acting only on the probe in $\boldsymbol{x}^{\star}$, hereafter called the tagged probe. Under such condition, Eq.(1.1) transforms to the following stochastic evolution equation

$$
\frac{\partial}{\partial t} \mathbf{h}(\boldsymbol{x}, t)=\int d^{d} x^{\prime} \Lambda\left(\boldsymbol{x}-\boldsymbol{x}^{\prime}\right)\left[\frac{\partial^{z}}{\partial\left|\boldsymbol{x}^{\prime}\right|^{z}} \mathbf{h}\left(\boldsymbol{x}^{\prime}, t\right)+\mathbf{F}\left\{\mathbf{h}\left(\boldsymbol{x}^{\prime}, t\right), t\right\} \delta\left(\boldsymbol{x}^{\prime}-\boldsymbol{x}^{\star}\right)\right]+\boldsymbol{\eta}(\boldsymbol{x}, t) .
$$

Here $\mathbf{F}\{\mathbf{h}(\boldsymbol{x}, t), t\}$ is a force functional of the stochastic fields $\mathbf{h}(\boldsymbol{x}, t)$ and of the time $t$ : it represents the external perturbation applied to the probe particle placed at the position $\boldsymbol{x}^{\star}$. We derived the FLE for the probes at positions $\boldsymbol{x}^{\star}$ and $\boldsymbol{x}$ (untagged probe). Our interest is motivated by the increasingly performing nano- and micro-manipulation techniques, that nowadays allow experimentalists to detect the (nano) microscopic fluctuations on a system where a localized external force is applied, as in the case of optical or magnetic tweezer acting on a beads attached to a membrane surface or to a single spot along the polymer backbone [46,47]. We show that the Fox $H$-function formalism constitutes an excellent tool for tackling the problem of the compact representation and the systematic analysis of the scaling properties of any correlation function and drift when a localized potential is applied to the GEM system (1.1). Moreover we demonstrate the usefulness of the Fox $H$-functions in recovering the Einstein and Kubo fluctuation relations.

This article is organized according to the following structure. In Section 2 we recall the results formerly obtained within the FLE framework in the absence of any applied force. Moreover, we derive the velocityvelocity and position-velocity correlation function in terms of the Fox $H$-functions. In Section 3, starting from the expression (1.5), we derive the FLE equation both for the tracer particle placed at position $\boldsymbol{x}^{\star}$ and for a particle at a generic position $\boldsymbol{x}$, we also study the scaling properties of the noise- and forcepropagators. In Section 4 we deal with the situation where the force applied to the probe particle in $\boldsymbol{x}^{\star}$ is constant: we study the scaling properties of the probe average drifts, show the validity of the Kubo fluctuation relations (KFR) and derive exact results for the Edwards-Wilkinson chain. In Appendix A we list the Fox $H$-functions properties that we use in our analysis.

\section{Fractional Langevin Equation framework}

In Ref. [1] we showed that the statistical properties of the stochastic systems governed by the equation (1.1) can be obtained within the framework of the following fractional Langevin equation for the probe's coordinate $h=h_{j}$ placed at position $\boldsymbol{x}$,

$$
K^{+}{ }_{-\infty} D_{t}^{\beta} h(\boldsymbol{x}, t)=\zeta(\boldsymbol{x}, t),
$$

where

$$
\begin{gathered}
\beta=\frac{2(z-d)}{\gamma}, \\
\gamma=2(z+\alpha-d),
\end{gathered}
$$

and

$$
K^{+}=\pi^{d / 2-1} \frac{\Gamma(d / 2) \sin (\pi \beta)(z+\alpha-d)}{2^{1-d} A^{\beta}} .
$$

The pseudo-differential operator 


$$
{ }_{a} D_{t}^{\beta} \phi(t)=\frac{1}{\Gamma(1-\beta)} \frac{d}{d t} \int_{a}^{t} d t^{\prime} \frac{1}{\left(t-t^{\prime}\right)^{\beta}} \phi\left(t^{\prime}\right), \quad 0<\beta<1,
$$

represents the left side Riemann-Liouville derivative with lower bound $a<t \quad[3,48]$. We remind that the derivative (2.5) is equivalent to the Caputo [49] derivative, i.e.

$$
\frac{d^{\beta}}{d t^{\beta}} X(t)=\frac{1}{\Gamma(1-\beta)} \int_{a}^{t} d t^{\prime} \frac{\dot{X}\left(t^{\prime}\right)}{\left(t-t^{\prime}\right)^{\beta}} \quad 0<\beta<1,
$$

if the lower bound in (2.5) is set $a=-\infty$ [48].

The tracer dynamical representation provided by the equation (2.1) is only valid whenever $z>d$, i.e. $\beta<1((d-1) / 2<\alpha<d)$. In the case of local hydrodynamics $(\Lambda(\boldsymbol{r})=\delta(|\boldsymbol{r}|))$ it is sufficient to set $\alpha=d$ and $A=$ const.

As shown in Ref. [31], in spite of their apparent difference, equations (1.1) and (2.1) provide the same level of accuracy in the description of the system dynamics. Indeed, while the Markovian stochastic behavior of the entire system is ruled by (1.1), Eq.(2.1) reproduces the tracer non-Markovian fractional Brownian motion. Nonetheless, the overall Markovian and the local non-Markovian representations coincide in furnishing the analytical expression of any system physical observable. This is evident by the properties of the fractional Gaussian noise (fGn) $\zeta(\boldsymbol{x}, t)$ appearing on the right hand side of $(2.1)$, which is correlated in time and in space [31],

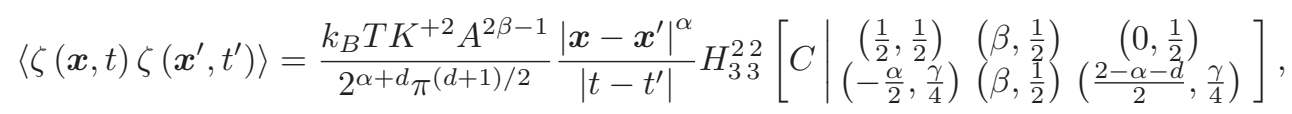

where we have introduced the Fox $H$-function [32-34], and defined

$$
C=\frac{2}{A\left|t-t^{\prime}\right|}\left(\frac{\left|\boldsymbol{x}-\boldsymbol{x}^{\prime}\right|}{2}\right)^{\frac{\gamma}{2}} .
$$

Setting $\boldsymbol{x} \rightarrow \boldsymbol{x}^{\prime}$ in Eq.(2.7) gives the fluctuation-dissipation relation, i.e.

$$
\left\langle\zeta(\boldsymbol{x}, t) \zeta\left(\boldsymbol{x}, t^{\prime}\right)\right\rangle=k_{B} T \frac{K^{+}}{\Gamma(1-\beta)\left|t-t^{\prime}\right|^{\beta}} .
$$

Moreover, thanks to Eqs.(2.1, 2.7), the two-point two-time correlation function of the stochastic field $h(\boldsymbol{x}, t)$ is expressed as [31]

$$
\begin{aligned}
& \left\langle h(\boldsymbol{x}, t) h\left(\boldsymbol{x}^{\prime}, t^{\prime}\right)\right\rangle=\frac{2 k_{B} T A^{2 \beta-1}}{2^{\alpha+d} \gamma \pi^{d / 2+1}}\left|\boldsymbol{x}-\boldsymbol{x}^{\prime}\right|^{\alpha} \int_{0}^{+\infty} d \omega \times \\
& \frac{\cos \left(\omega\left(t-t^{\prime}\right)\right)}{\omega^{2 \beta}} H_{13}^{21}\left[\begin{array}{l}
\frac{k}{2} \mid \begin{array}{l}
\left.\beta, \frac{1}{\gamma}\right) \\
\left(\frac{-\alpha}{2}, \frac{1}{2}\right)
\end{array}\left(\beta, \frac{1}{\gamma}\right)\left(\frac{2-\alpha-d}{2}, \frac{1}{2}\right)
\end{array}\right] .
\end{aligned}
$$

where

$$
k=\left(\frac{|\omega|}{A}\right)^{2 / \gamma}\left|\boldsymbol{x}-\boldsymbol{x}^{\prime}\right| .
$$

Now, we want to derive the velocity correlation functions, as arising from the FLE (2.1). Let us first introduce the Fourier transform of a function $\phi(\boldsymbol{x}, t)$ as

$$
\phi(\boldsymbol{q}, \omega)=\int_{-\infty}^{+\infty} d^{d} x \int_{-\infty}^{+\infty} d t \phi(\boldsymbol{x}, t) e^{-i(\boldsymbol{q} \cdot \boldsymbol{x}-\omega t)}
$$

and introduce the short notation for the time Fourier transform of the force: 


$$
\mathcal{F}_{\omega}\left\{\mathbf{F}\left\{\mathbf{f}\left(\boldsymbol{x}^{\star}, t\right), t\right\}\right\} \equiv \int_{-\infty}^{+\infty} d t \mathbf{F}\left\{\mathbf{f}\left(\boldsymbol{x}^{\star}, t\right), t\right\} e^{i \omega t} .
$$

Hence, it is immediate to prove that in the Fourier space

$$
\left\langle v(\boldsymbol{x}, \omega) v\left(\boldsymbol{x}^{\prime}, \omega^{\prime}\right)\right\rangle=\frac{1}{K^{+2}} \frac{\left\langle\zeta(\boldsymbol{x}, \omega) \zeta\left(\boldsymbol{x}^{\prime}, \omega^{\prime}\right)\right\rangle}{(-i \omega)^{\beta-1}\left(-i \omega^{\prime}\right)^{\beta-1}}
$$

Thanks to the Fourier transform of the noise correlation function (2.7) we can express the former definition as

$$
\begin{aligned}
& \left\langle v(\boldsymbol{x}, \omega) v\left(\boldsymbol{x}^{\prime}, \omega^{\prime}\right)\right\rangle=\frac{k_{B} T A^{2 \beta-1}}{2^{\alpha+d-2} \gamma \pi^{d / 2-1}}\left|\boldsymbol{x}-\boldsymbol{x}^{\prime}\right|^{\alpha}|\omega|^{2-2 \beta} \times \\
& \delta\left(\omega+\omega^{\prime}\right) H_{13}^{21}\left[\frac{k}{2} \mid \begin{array}{c}
\left(\beta, \frac{1}{\gamma}\right) \\
\left(-\frac{\alpha}{2}, \frac{1}{2}\right)
\end{array}\left(\begin{array}{l}
\left.\beta, \frac{1}{\gamma}\right) \\
\left(\frac{2-\alpha-d}{2}, \frac{1}{2}\right)
\end{array}\right] .\right.
\end{aligned}
$$

where $k$ has been defined in (2.11).

We note that the same expression can be furnished starting from the GEM (1.1). Indeed, the solution of (1.1) in the Fourier space for the unperturbed systems is

$$
h(\boldsymbol{q}, \omega)=\frac{\eta(\boldsymbol{q}, \omega)}{-i \omega+A|\boldsymbol{q}|^{\gamma / 2}},
$$

and for the velocity

$$
v(\boldsymbol{q}, \omega)=\frac{-i \omega \eta(\boldsymbol{q}, \omega)}{-i \omega+A|\boldsymbol{q}|^{\gamma / 2}} .
$$

Thanks to the noise properties in Fourier-Fourier space $\left\langle\eta(\boldsymbol{q}, \omega) \eta\left(\boldsymbol{q}^{\prime}, \omega^{\prime}\right)\right\rangle=2 k_{B} T \Lambda(|\boldsymbol{q}|)(2 \pi)^{d+1} \delta(\omega+$ $\left.\omega^{\prime}\right) \delta\left(\boldsymbol{q}+\boldsymbol{q}^{\prime}\right)$ we obtain

$$
\begin{aligned}
& \left\langle v(\boldsymbol{x}, \omega) v\left(\boldsymbol{x}^{\prime}, \omega^{\prime}\right)\right\rangle=\frac{4 \pi k_{B} T \delta\left(\omega+\omega^{\prime}\right) A\left|\boldsymbol{x}-\boldsymbol{x}^{\prime}\right|^{1-d / 2}}{(2 \pi)^{d / 2}} \times \\
& \quad \int_{0}^{+\infty} d|\boldsymbol{q}||\boldsymbol{q}|^{\alpha-d / 2} J_{d / 2-1}\left(|\boldsymbol{q}|\left|\boldsymbol{x}-\boldsymbol{x}^{\star}\right|\right) \frac{\omega^{2}}{\omega^{2}+A^{2}|\boldsymbol{q}|^{\gamma}},
\end{aligned}
$$

where in addition, we made use of the definition of the $d$-dimensional inverse Fourier transform of an isotropic function $\phi(|\boldsymbol{q}|)$ [50]

$$
\int_{-\infty}^{+\infty} \frac{d^{d} q}{(2 \pi)^{d}} e^{i \boldsymbol{q} \cdot \boldsymbol{r}} \phi(|\boldsymbol{q}|)=\frac{|\boldsymbol{r}|^{1-d / 2}}{(2 \pi)^{d / 2}} \int_{0}^{+\infty} d|\boldsymbol{q}||\boldsymbol{q}|^{d / 2} J_{d / 2-1}(|\boldsymbol{q}||\boldsymbol{r}|) \phi(|\boldsymbol{q}|)
$$

with $J_{d / 2-1}$ the Bessel function of fractional order $d / 2-1$. Hence, applying the change of variable $y=A^{2 / \gamma}|\boldsymbol{q}|$ and recalling that [31],

$$
\frac{1}{1+y^{\delta}}=\frac{1}{\delta} H_{11}^{11}\left[y \mid \begin{array}{c}
\left(1, \frac{1}{\delta}\right) \\
\left(1, \frac{1}{\delta}\right)
\end{array}\right],
$$

after employing the property (A.10) we get the expression (2.15).

Now we want to invert Eq. (2.15) in the time domain, i.e.

$$
\begin{aligned}
& \left\langle v(\boldsymbol{x}, t) v\left(\boldsymbol{x}^{\prime}, t^{\prime}\right)\right\rangle=\frac{k_{B} T A^{2 \beta-1}}{2^{\alpha+d-1} \gamma \pi^{d / 2+1}}\left|\boldsymbol{x}-\boldsymbol{x}^{\prime}\right|^{\alpha} \int_{0}^{\infty} d \omega \times \\
& \cos \left(\omega\left|t-t^{\prime}\right|\right) \omega^{2-2 \beta} H_{13}^{21}\left[\frac{k}{2} \mid \begin{array}{c}
\left(\beta, \frac{1}{\gamma}\right) \\
\left(-\frac{\alpha}{2}, \frac{1}{2}\right)\left(\beta, \frac{1}{\gamma}\right)\left(\frac{2-\alpha-d}{2}, \frac{1}{2}\right)
\end{array}\right] .
\end{aligned}
$$


Note that the integral here does not present any divergence in the limit $\omega \rightarrow 0$ as instead of Eq.(2.10).

This can be checked by expanding the Fox $H$-function for small argument $k$ thanks to (A.13)

$$
H_{13}^{21}\left[\frac{k}{2} \mid\left(-\frac{\alpha}{2}, \frac{1}{2}\right)\left(\beta, \frac{1}{\gamma}\right)\left(\frac{2-\alpha-d}{2}, \frac{1}{2}\right)\right] \simeq \frac{2 \pi}{\sin (\alpha \pi / \gamma) \Gamma(d / 2)} k^{-\alpha} .
$$

Inserting the previous expansion in (2.21) one clearly sees that the power inside the integral is $\propto \omega^{2 \alpha / \gamma}$, so that we can proceed to evaluate the expression (2.21). Making use of (A.6) we arrive at the expression:

$$
\left\langle v(\boldsymbol{x}, t) v\left(\boldsymbol{x}^{\prime}, t^{\prime}\right)\right\rangle=\frac{k_{B} T A^{2 \beta-1}}{2^{\alpha+d-2+2 \beta} \pi^{(d+1) / 2}} \frac{\left|\boldsymbol{x}-\boldsymbol{x}^{\prime}\right|^{\alpha}}{\left|t-t^{\prime}\right|^{3-2 \beta}} H_{33}^{22}\left[\begin{array}{c|c}
\left(-\frac{1}{2}+\beta, \frac{1}{2}\right)\left(\beta, \frac{1}{2}\right)\left(-1+\beta, \frac{1}{2}\right) \\
\left(-\frac{\alpha}{2}, \frac{\gamma}{4}\right) \quad\left(\beta, \frac{1}{2}\right)\left(\frac{2-\alpha-d}{2}, \frac{\gamma}{4}\right)
\end{array}\right],
$$

where $C$ has been defined in (2.8). Manipulating the previous form through (A.8) and (A.9) one arrives at

$$
\left\langle v(\boldsymbol{x}, t) v\left(\boldsymbol{x}^{\prime}, t^{\prime}\right)\right\rangle=\frac{k_{B} T A}{2^{d-\alpha} \pi^{(d+1) / 2}} \frac{\left|\boldsymbol{x}-\boldsymbol{x}^{\prime}\right|^{-\alpha}}{\left|t-t^{\prime}\right|} H_{33}^{22}\left[C \mid \begin{array}{c}
\left(\frac{1}{2}, \frac{1}{2}\right) \\
\left(\frac{\alpha}{2}, \frac{\gamma}{4}\right)
\end{array}\left(\begin{array}{c}
\left.1, \frac{1}{2}\right) \\
\left(1, \frac{1}{2}\right) \\
\left(1+\frac{\alpha-d}{2}, \frac{\gamma}{4}\right)
\end{array}\right],\right.
$$

If we want to calculate the autocorrelation function we must set $\boldsymbol{x} \rightarrow \boldsymbol{x}^{\prime}$. Expanding for small $k$ the Fox $H$-function in (2.24) by means of (A.13), we arrive at the expression

$$
\left\langle v(\boldsymbol{x}, t) v\left(\boldsymbol{x}, t^{\prime}\right)\right\rangle=-\frac{\alpha k_{B} T A^{\beta} 2^{3-d}}{\gamma^{2} \pi^{d / 2}} \frac{\Gamma(1-\beta)}{\Gamma(d / 2)\left|t-t^{\prime}\right|^{2-\beta}},
$$

from which it is found that the field velocity is always anti-correlated, as it is expected for subdiffusive fractional Brownian systems [22].

The next step that we want to take is the calculation of the position-velocity correlation function $\left\langle h(\boldsymbol{x}, t) v\left(\boldsymbol{x}^{\prime}, t^{\prime}\right)\right\rangle$. We do it within the framework of GEM (1.1). From (2.16), (2.17) and (2.19) we achieve

$$
\left\langle h(\boldsymbol{x}, t) v\left(\boldsymbol{x}^{\prime}, t^{\prime}\right)\right\rangle=\frac{2 k_{B} T A\left|\boldsymbol{x}-\boldsymbol{x}^{\star}\right|^{1-d / 2}}{(2 \pi)^{d / 2}} \int_{0}^{+\infty} d|\boldsymbol{q}||\boldsymbol{q}|^{\alpha-d / 2} J_{d / 2-1}\left(|\boldsymbol{q}|\left|\boldsymbol{x}-\boldsymbol{x}^{\prime}\right|\right) \int_{-\infty}^{+\infty} \frac{d \omega}{2 \pi} \frac{i \omega e^{-i \omega\left|t-t^{\prime}\right|}}{A^{2}|\boldsymbol{q}|^{\gamma}+\omega^{2}}
$$

which, inverted in time, gives

$$
\left\langle h(\boldsymbol{x}, t) v\left(\boldsymbol{x}^{\prime}, t^{\prime}\right)\right\rangle=\frac{k_{B} T A\left|\boldsymbol{x}-\boldsymbol{x}^{\star}\right|^{1-d / 2}}{(2 \pi)^{d / 2}} \int_{0}^{+\infty} d|\boldsymbol{q}||\boldsymbol{q}|^{\alpha-d / 2} J_{d / 2-1}\left(|\boldsymbol{q}|\left|\boldsymbol{x}-\boldsymbol{x}^{\prime}\right|\right) e^{-A|\boldsymbol{q}|^{\gamma / 2}\left|t-t^{\prime}\right|}
$$

Recalling that the exponential function has an $H$-representation which is

$$
e^{-y}=H_{01}^{10}\left[y \mid \begin{array}{c}
- \\
(0,1)
\end{array}\right] \text {, }
$$

and using the property (A.10), the Fox $H$-function expression for the above correlation function is

$$
\left\langle h(\boldsymbol{x}, t) v\left(\boldsymbol{x}^{\star}, t^{\prime}\right)\right\rangle=\frac{k_{B} T A\left|\boldsymbol{x}-\boldsymbol{x}^{\star}\right|^{-\alpha}}{2^{d-\alpha} \pi^{d / 2}} H_{21}^{11}\left[A\left|t-t^{\prime}\right|\left(\frac{2}{\left|\boldsymbol{x}-\boldsymbol{x}^{\star}\right|}\right)^{\gamma / 2} \mid \begin{array}{c}
\left(1-\frac{\alpha}{2}, \frac{\gamma}{4}\right)\left(\frac{d-\alpha}{2}, \frac{\gamma}{4}\right) \\
(0,1)
\end{array}\right] .
$$




\section{Fractional Langevin Equation with applied force}

For consistency, we hereby remind briefly the derivation of the FLE for the probe particle placed both at the position $\boldsymbol{x}^{\star}$ (tagged probe), where the external force is applied, and at the generic position $\boldsymbol{x}$ (untaged probe) [45].

In the Fourier space the solution of (1.5) is obtained as

$$
\mathbf{h}(\boldsymbol{q}, \omega)=\frac{A \mathcal{F}_{\omega}\left\{\mathbf{F}\left\{\mathbf{h}\left(\boldsymbol{x}^{\star}, t\right), t\right\}\right\} e^{-i \boldsymbol{q} \cdot \boldsymbol{x}^{\star}}}{|\boldsymbol{q}|^{d-\alpha}\left(-i \omega+A|\boldsymbol{q}|^{\gamma / 2}\right)}+\frac{\boldsymbol{\eta}(\boldsymbol{q}, \omega)}{-i \omega+A|\boldsymbol{q}|^{\gamma / 2}},
$$

where we made use of the definition (1.3). We then multiply both sides of $(3.1)$ by $K^{+}(-i \omega)^{\beta}$, where $K^{+}$and $\beta$ have been defined respectively in (2.4) and (2.2):

$$
K^{+}(-i \omega)^{\beta} \mathbf{h}(\boldsymbol{q}, \omega)=\mathcal{F}_{\omega}\left\{\mathbf{F}\left\{\mathbf{h}\left(\boldsymbol{x}^{\star}, t\right), t\right\}\right\} \frac{A K^{+}(-i \omega)^{\beta} e^{-i \boldsymbol{q} \cdot x^{\star}}}{|\boldsymbol{q}|^{d-\alpha}\left(-i \omega+A|\boldsymbol{q}|^{\gamma / 2}\right)}+\frac{K^{+}(-i \omega)^{\beta}}{-i \omega+A|\boldsymbol{q}|^{\gamma / 2}} \boldsymbol{\eta}(\boldsymbol{q}, \omega) .
$$

We now derive the FLE for the tracer at a generic position $x$ : to proceed further we first have to invert in the space domain both terms on the right side of (3.2). For the first we get

$$
\begin{aligned}
& \mathcal{F}_{\omega}\left\{\mathbf{F}\left\{\mathbf{h}\left(\boldsymbol{x}^{\star}, t\right), t\right\}\right\} A K^{+}(-i \omega)^{\beta} \int_{-\infty}^{+\infty} \frac{d^{d} q}{(2 \pi)^{d}} \frac{e^{i \boldsymbol{q} \cdot\left(\boldsymbol{x}-\boldsymbol{x}^{\star}\right)}}{|\boldsymbol{q}|^{d-\alpha}\left(-i \omega+A|\boldsymbol{q}|^{\gamma / 2}\right)}= \\
& \mathcal{F}_{\omega}\left\{\mathbf{F}\left\{\mathbf{h}\left(\boldsymbol{x}^{\star}, t\right), t\right\}\right\} \frac{A K^{+}(-i \omega)^{\beta}\left|\boldsymbol{x}-\boldsymbol{x}^{\star}\right|^{1-d / 2}}{(2 \pi)^{d / 2}} \int_{0}^{+\infty} d|\boldsymbol{q}| \frac{|\boldsymbol{q}|^{\alpha-d / 2} J_{d / 2-1}\left(|\boldsymbol{q}|\left|\boldsymbol{x}-\boldsymbol{x}^{\star}\right|\right)}{-i \omega+A|\boldsymbol{q}|^{\gamma / 2}} .
\end{aligned}
$$

Defining the following function as

$$
\Theta(|\boldsymbol{x}|, \omega)=\frac{A K^{+}(-i \omega)^{\beta}|\boldsymbol{x}|^{1-d / 2}}{(2 \pi)^{d / 2}} \int_{0}^{+\infty} d|\boldsymbol{q}| \frac{|\boldsymbol{q}|^{\alpha-d / 2} J_{d / 2-1}(|\boldsymbol{q}||\boldsymbol{x}|)}{-i \omega+A|\boldsymbol{q}|^{\gamma / 2}},
$$

we obtain that the first term in the right hand side of Eq.(3.2) after the inverse Fourier transform in space is given by

$$
\mathcal{F}_{\omega}\left\{\mathbf{F}\left\{\mathbf{h}\left(\boldsymbol{x}^{\star}, t\right), t\right\}\right\} \Theta\left(\left|\boldsymbol{x}-\boldsymbol{x}^{\star}\right|, \omega\right) .
$$

The second term can be treated in the same way $[1,31]$. Indeed, inverting in the space domain we find

$$
\boldsymbol{\zeta}(\boldsymbol{x}, \omega)=\int_{-\infty}^{+\infty} d \boldsymbol{x}^{\prime} \boldsymbol{\eta}\left(\boldsymbol{x}^{\prime}, \omega\right) \Phi\left(\left|\boldsymbol{x}^{\prime}-\boldsymbol{x}\right|, \omega\right)
$$

where, according to (2.19), the function $\Phi(|\boldsymbol{x}|, \omega)$ is defined as

$$
\Phi(|\boldsymbol{x}|, \omega)=\frac{K^{+}(-i \omega)^{\beta}|\boldsymbol{x}|^{1-d / 2}}{(2 \pi)^{d / 2}} \int_{0}^{+\infty} d|\boldsymbol{q}| \frac{|\boldsymbol{q}|^{d / 2} J_{d / 2-1}(|\boldsymbol{q}||\boldsymbol{x}|)}{-i \omega+A|\boldsymbol{q}|^{\gamma / 2}} .
$$

By combining Eqs.(3.5) and (3.6) we can write Eq.(3.2) after its invertion in the space domain as

$$
K^{+}(-i \omega)^{\beta} \mathbf{h}(\boldsymbol{x}, \omega)=\mathcal{F}_{\omega}\left\{\mathbf{F}\left\{\mathbf{h}\left(\boldsymbol{x}^{\star}, t\right), t\right\}\right\} \Theta\left(\left|\boldsymbol{x}-\boldsymbol{x}^{\star}\right|, \omega\right)+\boldsymbol{\zeta}(\boldsymbol{x}, \omega)
$$

It is convenient to use Eq.(3.8) as a starting point to calculate the response for the untagged and tagged probes in the subsequent Section.

The inverse Fourier transforms of $\Theta(|\boldsymbol{x}|, \omega)$ and $\Phi(|\boldsymbol{x}|, \omega)$ are performed as follows. Let us start from the force propagator $\Theta(|\boldsymbol{x}|, t)$. The key point here is the representation of the Riemann-Liouville fractional derivative (2.5) in Fourier space [48],

$$
\int_{-\infty}^{+\infty} d t e^{i \omega t}{ }_{-\infty} D_{t}^{\beta} \phi(t)=(-i \omega)^{\beta} \phi(\omega)
$$


Hence we invert in time the expression (3.4) as

$$
\Theta(|\boldsymbol{x}|, t)=\frac{A K^{+}|\boldsymbol{x}|^{1-d / 2}}{(2 \pi)^{d / 2}} \int_{0}^{+\infty} d|\boldsymbol{q}||\boldsymbol{q}|^{\alpha-d / 2} J_{d / 2-1}(|\boldsymbol{q}||\boldsymbol{x}|)_{-\infty} D_{t}^{\beta}\left(e^{-A|\boldsymbol{q}|^{\gamma / 2} t} \theta(t)\right) .
$$

where $\theta(t)$ represents the Heaviside step function.

From Eq.(3.7) it results that the noise-propagator $\Phi(|\boldsymbol{x}|, t)$ has the same structure as $\Theta(|\boldsymbol{x}|, t)$. We can then treat it in the same way, obtaining

$$
\Phi(|\boldsymbol{x}|, t)=\frac{A K^{+}|\boldsymbol{x}|^{1-d / 2}}{(2 \pi)^{d / 2}} \int_{0}^{+\infty} d|\boldsymbol{q}||\boldsymbol{q}|^{d / 2} J_{d / 2-1}(|\boldsymbol{q}||\boldsymbol{x}|)_{-\infty} D_{t}^{\beta}\left(e^{-A|\boldsymbol{q}|^{\gamma / 2} t} \theta(t)\right) .
$$

Taking into account the definition (2.5) we rewrite Eqs.(3.10) and (3.11) as

$$
\Theta(|\boldsymbol{x}|, t)=\frac{A K^{+}|\boldsymbol{x}|^{1-d / 2}}{(2 \pi)^{d / 2}}{ }_{0} D_{t}^{\beta} \int_{0}^{+\infty} d|\boldsymbol{q}||\boldsymbol{q}|^{\alpha-d / 2} J_{d / 2-1}(|\boldsymbol{q}||\boldsymbol{x}|) e^{-A|\boldsymbol{q}|^{\gamma / 2} t},
$$

and

$$
\Phi(|\boldsymbol{x}|, t)=\frac{A K^{+}|\boldsymbol{x}|^{1-d / 2}}{(2 \pi)^{d / 2}}{ }_{0} D_{t}^{\beta} \int_{0}^{+\infty} d|\boldsymbol{q}||\boldsymbol{q}|^{d / 2} J_{d / 2-1}(|\boldsymbol{q}||\boldsymbol{x}|) e^{-A|\boldsymbol{q}|^{\gamma / 2} t},
$$

where ${ }_{0} D_{t}^{\beta}$ represents the Riemann-Liouville fractional derivative (2.5) with the lower bound $a=0$. In Eqs.(3.12) and (3.13) $t$ is non-negative, otherwise Eqs.(3.10) and (3.11) give zero. Furthermore we note that the two integral expressions (3.12) and (3.13) coincide in the special case of local hydrodynamic interactions.

The two propagators can be expressed in terms of the Fox $H$-functions by the use of Eq.(2.28), the property (A.10) and the fractional derivative of the Fox $H$-function (A.11) [35]:

$$
\Theta(|\boldsymbol{x}|, t)=\frac{A^{1+\beta} K^{+} 2^{\gamma / 2-d}}{\pi^{d / 2}|\boldsymbol{x}|^{\gamma / 2}} H_{32}^{12}\left[2^{\gamma / 2} \frac{t}{\tau(|\boldsymbol{x}|)} \mid \begin{array}{cc}
(-\beta, 1) \\
(-\beta, 1)
\end{array}\left(\begin{array}{c}
\left(1-\frac{\gamma}{4}, \frac{\gamma}{4}\right)\left(\frac{2 d-z-\alpha}{2}, \frac{\gamma}{4}\right) \\
(0,1)
\end{array}\right] .\right.
$$

and

$$
\Phi(|\boldsymbol{x}|, t)=\frac{A^{1+\beta} K^{+} 2^{z-d}}{\pi^{d / 2}|\boldsymbol{x}|^{z}} H_{32}^{12}\left[\begin{array}{ll}
2^{\gamma / 2} \frac{t}{\tau(|\boldsymbol{x}|)} \mid \begin{array}{c}
(-\beta, 1) \\
(-\beta, 1)
\end{array} & \left(1-\frac{z}{2}, \frac{\gamma}{4}\right)\left(\frac{d-z}{2}, \frac{\gamma}{4}\right)
\end{array}\right]
$$

where the correlation time has been introduced as $[45,51]$

$$
\tau(|\boldsymbol{x}|)=\frac{|\boldsymbol{x}|^{\gamma / 2}}{A} .
$$

Finally, applying the inverse Fourier transformation in time to Eq. (3.8), we get the form of the FLE for the particle at a generic position $\boldsymbol{x}$ :

$$
K^{+}{ }_{-\infty} D_{t}^{\beta} \mathbf{h}(\boldsymbol{x}, t)=\int_{-\infty}^{t} d t^{\prime} \mathbf{F}\left\{\mathbf{h}\left(\boldsymbol{x}^{\star}, t^{\prime}\right), t^{\prime}\right\} \Theta\left(\left|\boldsymbol{x}-\boldsymbol{x}^{\star}\right|, t-t^{\prime}\right)+\boldsymbol{\zeta}(\boldsymbol{x}, t),
$$

where the non-Markovian noise

$$
\boldsymbol{\zeta}(\boldsymbol{x}, t)=\int_{-\infty}^{+\infty} d \boldsymbol{x}^{\prime} \int_{-\infty}^{t} d t^{\prime} \boldsymbol{\eta}\left(\boldsymbol{x}^{\prime}, t^{\prime}\right) \Phi\left(\left|\boldsymbol{x}^{\prime}-\boldsymbol{x}\right|, t-t^{\prime}\right)
$$

fulfills fluctuation-dissipation relation (2.9). The analysis of (3.17) shows that the probe particle at the position $\boldsymbol{x}$ undergoes an effective force that, compared to the force acting on $\boldsymbol{x}^{\star}$, is shifted in time 
and in space according to the function defined in (3.14). Indeed, $\Theta\left(\left|\boldsymbol{x}-\boldsymbol{x}^{\star}\right|, t-t^{\prime}\right)$ can be seen as the propagator carrying the external perturbation exerted at the point $\boldsymbol{x}^{\star}$ at time $t^{\prime}$, to the point $\boldsymbol{x}$ at time $t$. Likewise, the function $\Phi\left(\left|\boldsymbol{x}-\boldsymbol{x}^{\star}\right|, t-t^{\prime}\right)$ represents the propagator of the Brownian random source $\boldsymbol{\eta}(\boldsymbol{x}, t)$ from the point $\boldsymbol{x}^{\prime}$ to the point $\boldsymbol{x}$ in the time elapsed between $t^{\prime}$ and $t$ : the sum of the contributions arising from the whole system within the interval $t^{\prime} \in[-\infty, t]$ generates stationary non-Markovian fGn $\boldsymbol{\zeta}(\boldsymbol{x}, t)$.

We point out that in Eqs.(3.17) and (3.18) time integrals extend to $t$ instead of $+\infty$ as formally required by the definition of the inverse Fourier transform. Indeed, both propagators $\Theta(|\boldsymbol{x}|, t)$ and $\Phi(|\boldsymbol{x}|, t)$ differ from zero only for $t>0$, as it was already mentioned. Moreover, equations (3.14) and (3.15) highlight the valuable property for which the propagators attain different regimes whether the time $t$ is larger or smaller than the characteristic correlation time $\tau$. As a matter of fact, the Fox $H$-function formalism provides a comprehensive mathematical formulation of a function showing double scaling behavior, as any Fox $H$-function exhibits different regimes for small and large values of its argument [43, 44]. The time $\tau(|\boldsymbol{x}|)$ can be interpreted as the time needed for the information (either an external perturbation or the same random source $\boldsymbol{\eta}$ ) to travel within a distance $|\boldsymbol{x}|$ along the elastic system [45,51]. Similarly, the correlation length $\xi(t)=(A t)^{2 / \gamma}$ represents the distance within which the points are mutually influencing their stochastic dynamics $[14,15,52-54]$. The two scaling regimes exhibited by the propagators (3.14) and (3.15) have strong implications in the two-point two-time correlation functions behavior [51] and, most important, in the probes responses to external perturbation, as demonstrated below.

We now turn to the derivation of the FLE for the tagged tracer at $\boldsymbol{x}^{\star}$. In this case it is sufficient to take the limit $\boldsymbol{x} \rightarrow \boldsymbol{x}^{\star}$ in (3.17). Taking expression (3.4), we recall that the Bessel function expansion for small argument is [55]

$$
J_{d / 2-1}(r) \sim \frac{1}{\Gamma(d / 2)}\left(\frac{2}{r}\right)^{1-d / 2}
$$

from which we have

$$
\Theta(0, \omega)=\frac{A K^{+}(-i \omega)^{\beta}}{2^{d-1}(\pi)^{d / 2} \Gamma(d / 2)} \int_{0}^{+\infty} d|\boldsymbol{q}| \frac{|\boldsymbol{q}|^{\alpha-1}}{-i \omega+A|\boldsymbol{q}|^{\gamma / 2}} .
$$

Changing variable $y=|\boldsymbol{q}|^{\gamma / 2}$, solving the integral according to Ref. [56], and thanks to the definition of $K^{+}(2.4)$ we obtain that

$$
\Theta(0, \omega)=1
$$

which, substituted in (3.5) and inverted in time, gives the FLE expression for the probe particle placed at $\boldsymbol{x}^{\star}$ :

$$
K^{+} D_{C}^{\beta} \mathbf{h}\left(\boldsymbol{x}^{\star}, t\right)=\mathbf{F}\left\{\mathbf{h}\left(\boldsymbol{x}^{\star}, t\right), t\right\}+\boldsymbol{\zeta}\left(\boldsymbol{x}^{\star}, t\right) .
$$

\section{Constant force: $F_{0} \theta(t)$}

The situation that we are going to address in this paper concerns an external constant force applied to the tagged probe $\boldsymbol{x}^{\star}$, i.e.

$$
\mathbf{F}\left\{\mathbf{h}\left(\boldsymbol{x}^{\star}, t\right), t\right\}=F_{0} \theta(t) .
$$

where $F_{0}$ represents the force along one direction (say $F_{0} \equiv F_{j}$ ) and $\theta(t)$ is the Heaviside step function. We are interested in the average drift of the tagged and untagged tracers in $\boldsymbol{x}$ and $\boldsymbol{x}^{\star}$, namely $\langle h(\boldsymbol{x}, t)\rangle_{F_{0}}$ and $\left\langle h\left(\boldsymbol{x}^{\star}, t\right)\right\rangle_{F_{0}}$ respectively. We first focus on the untagged probe. The starting point is Eq.(3.8), where the Fourier transform of the force is given by [57] 


$$
\mathcal{F}_{\omega}\left\{F_{0} \theta(t)\right\}=F_{0}\left\{\frac{i}{\omega}+\pi \delta(\omega)\right\}
$$

while $\Theta$ and $\boldsymbol{\zeta}$ are given by Eqs.(3.4) and (3.6), respectively. Using the fact that $\delta(\omega) \Theta(|\boldsymbol{x}|, \omega)=0$, we get the following equation for the Fourier transform of the average drift in time,

$$
-i \omega\langle h(\boldsymbol{x}, \omega)\rangle_{F_{0}}=\frac{A|\boldsymbol{x}|^{1-d / 2}}{(2 \pi)^{d / 2}} F_{0} \int_{0}^{+\infty} d|\boldsymbol{q}| \frac{|\boldsymbol{q}|^{\alpha-d / 2} J_{d / 2-1}(|\boldsymbol{q}||\boldsymbol{x}|)}{-i \omega+A|\boldsymbol{q}|^{\gamma / 2}}
$$

Taking an inverse Fourier transform in time we get

$$
\frac{d\langle h(\boldsymbol{x}, t)\rangle_{F_{0}}}{d t}=\frac{A\left|\boldsymbol{x}-\boldsymbol{x}^{\star}\right|^{1-d / 2}}{(2 \pi)^{d / 2}} F_{0} \int_{0}^{+\infty} d|\boldsymbol{q}||\boldsymbol{q}|^{\alpha-d / 2} J_{d / 2-1}\left(|\boldsymbol{q}|\left|\boldsymbol{x}-\boldsymbol{x}^{\star}\right|\right) e^{-A|\boldsymbol{q}|^{\gamma / 2} t} \theta(t) .
$$

Integrating both sides of Eq.(4.4) from 0 to $t$ and noting that $\langle h(\boldsymbol{x}, t=0)\rangle_{F_{0}}=0$, we arrive at the following expression for the average drift,

$$
\langle h(\boldsymbol{x}, t)\rangle_{F_{0}}=\frac{A\left|\boldsymbol{x}-\boldsymbol{x}^{\star}\right|^{1-d / 2}}{(2 \pi)^{d / 2}} F_{0} \int_{0}^{t} d t^{\prime} \int_{0}^{+\infty} d|\boldsymbol{q}||\boldsymbol{q}|^{\alpha-d / 2} J_{d / 2-1}\left(|\boldsymbol{q}|\left|\boldsymbol{x}-\boldsymbol{x}^{\star}\right|\right) e^{-A|\boldsymbol{q}|^{\gamma / 2} t^{\prime}} .
$$

First we cast the exponential according to (2.28),

$$
\begin{aligned}
& \langle h(\boldsymbol{x}, t)\rangle_{F_{0}}= \\
& \frac{A\left|\boldsymbol{x}-\boldsymbol{x}^{\star}\right|^{1-d / 2}}{(2 \pi)^{d / 2}} F_{0} \int_{0}^{t} d t^{\prime} \int_{0}^{+\infty} d|\boldsymbol{q}||\boldsymbol{q}|^{\alpha-d / 2} J_{d / 2-1}\left(|\boldsymbol{q}|\left|\boldsymbol{x}-\boldsymbol{x}^{\star}\right|\right) H_{01}^{10}\left[A|\boldsymbol{q}|^{\alpha+z-d} t^{\prime} \mid \begin{array}{c}
- \\
(0,1)
\end{array}\right] .
\end{aligned}
$$

Thanks to the integral (A.10) it gets the form



Finally, after taking the integral over $t^{\prime}$ using (A.7), we get

$$
\langle h(\boldsymbol{x}, t)\rangle_{F_{0}}=\frac{\left|\boldsymbol{x}-\boldsymbol{x}^{\star}\right|^{z-d}}{2^{z} \pi^{d / 2}} F_{0} H_{32}^{12}\left[2^{\gamma / 2} \frac{t}{\tau\left(\left|\boldsymbol{x}-\boldsymbol{x}^{\star}\right|\right)} \mid \begin{array}{c}
\left.(1,1)\left(1+\frac{z-d}{2}, \frac{\gamma}{4}\right)\left(\frac{z}{2}, \frac{\gamma}{4}\right)\right] . \\
(1,1)(0,1)
\end{array}\right] .
$$

Taking here the limit $\boldsymbol{x} \rightarrow \boldsymbol{x}^{\star}$ and using the expansion (A.14) we arrive at the expression for the tagged probe drift,

$$
\left\langle h\left(\boldsymbol{x}^{\star}, t\right)\right\rangle_{F_{0}} \simeq \frac{2^{1-d / 2} A^{\beta} \Gamma(1-\beta)}{(2 \pi)^{d / 2} \Gamma(d / 2)(z-d)} F_{0} t^{\beta},
$$

where $\beta$ is given by Eq.(2.2). The motion of the untagged particle is more interesting, indeed its average drift attains two differrent regimes whether $t \ll \tau$ or $t \gg \tau$ respectively, in correspondence with the short and long time behavior of the Fox function appearing in Eq.(4.8). In the upcoming subsection we study the behaviour for short and long times of systems presenting long-range and local hydrodynamic interactions respectively. 


\section{1. $\langle h(x, t)\rangle_{F_{0}}$ scaling behavior}

Firstly, our analysis will concern with long range hydrodynamic systems.

- $\mathbf{t} \ll \boldsymbol{\tau}$. We start by using the Fox $H$-function expression (4.8): by expanding the Fox $H$-function according to (A.13) we obtain that for time shorter than correlation time $\tau$ the drift is given by

$$
\langle h(\boldsymbol{x}, t)\rangle_{F_{0}} \simeq \frac{A\left|\boldsymbol{x}-\boldsymbol{x}^{\star}\right|^{-\alpha}}{2^{d-\alpha} \pi^{d / 2}} \frac{\Gamma(\alpha / 2)}{\Gamma\left(\frac{d-\alpha}{2}\right)} F_{0} t .
$$

$-\mathbf{t} \gg \boldsymbol{\tau}$. The long time limit of the untagged probe average can be obtained by expanding (4.8) with the use of (A.14):

$$
\langle h(\boldsymbol{x}, t)\rangle_{F_{0}} \simeq\left\langle h\left(\boldsymbol{x}^{\star}, t\right)\right\rangle_{F_{0}} \simeq \frac{2^{1-d / 2} A^{\beta} \Gamma(1-\beta)}{(2 \pi)^{d / 2} \Gamma(d / 2)(z-d)} F_{0} t^{\beta}
$$

Now we consider local hydrodynamic interactions, hence we set $A=$ const, $\alpha=d, \gamma=2 z$ and $\beta=\frac{z-d}{z}$ in (4.8).

$-\mathbf{t} \ll \boldsymbol{\tau}$. An expansion of (4.8) according to (A.13) gives, when $z \neq 2 m(m \in \mathbb{N})$,

$$
\langle h(\boldsymbol{x}, t)\rangle_{F_{0}} \simeq \frac{2^{z-2} \Gamma\left(\frac{z+d}{2}\right) \Gamma\left(\frac{z}{2}\right)}{\pi^{d / 2+1}} \frac{z \sin \left(\frac{z \pi}{2}\right)}{\left|\boldsymbol{x}-\boldsymbol{x}^{\star}\right|^{z+d}} F_{0}(A t)^{2}, .
$$

Equation(4.12) points out that for $2+4 m<z<4+4 m$ the response of the probe is opposite to the external disturbance $F_{0}$, while for $4 m<z<2+4 m$ they have the same sign [45]. When $z=2 m$ the response is slower than any power so that we expect $[17,51]$

$$
\langle h(\boldsymbol{x}, t)\rangle_{F_{0}} \propto F_{0} \frac{t^{\beta+1}}{\left|\boldsymbol{x}-\boldsymbol{x}^{\star}\right|^{z}} e^{-\frac{\left|\boldsymbol{x}-\boldsymbol{x}^{\star}\right|^{1 / \beta}}{(A t)^{1 /(z-d)}}} .
$$

- $\mathbf{t} \gg \boldsymbol{\tau}$. For long times we can expand the expression (4.8) and get the same results as for long-range hydrodynamic system, see Eq.(4.11).

We can summarize the results obtained in this section in the following compact form

$$
\langle h(x, t)\rangle_{F_{0}}=F_{0} \frac{\left|\boldsymbol{x}-\boldsymbol{x}^{\star}\right|^{z-d}}{(2 \pi)^{d / 2}} f\left[\frac{t}{\tau\left(\left|\boldsymbol{x}-\boldsymbol{x}^{\star}\right|\right)}\right]
$$

The scaling function $f[u]$ exhibits two distinct behaviours whether $u \ll 1$ or $u \gg 1$. From (4.10), (4.12) and (4.13), it turns out that when $u \ll 1$

$$
f[u]\left\{\begin{array}{cc}
\sim 2^{\alpha-d / 2} \frac{\Gamma\left(\frac{\alpha}{2}\right)}{\Gamma\left(\frac{d-\alpha}{2}\right)} u & i) \\
\sim \frac{2^{z+d / 2-2}}{\pi} z \sin \left(\frac{z \pi}{2}\right) \Gamma\left(\frac{z}{2}\right) \Gamma\left(\frac{z+d}{2}\right) u^{2} & i i) \\
\propto u^{\beta+1} e^{-u^{1 /(d-z)}} & i i i)
\end{array}\right.
$$

for $i$ ) long range, $i i)$ local $(z \neq 2 m)$ and $i i i)$ local $(z=2 m)$ hydrodynamic interactions, respectively. When $u \gg 1$ we have invariably

$$
f[u] \simeq \frac{2^{1-d / 2}}{z-d} \frac{\Gamma(1-\beta)}{\Gamma\left(\frac{d}{2}\right)} u^{\beta} .
$$




\subsection{Kubo fluctuation relations}

In this subsection we show how the previous results satisfy the Kubo fluctuation relations [45, 59, 60] which connect the average drift in presence of an external disturbance $F_{0}$, to the correlation function in absence of any. Firstly we introduce the two-point two-time correlation function as

$$
\begin{aligned}
& \left\langle\delta_{t} h(\boldsymbol{x}, t) \delta_{t^{\prime}} h\left(\boldsymbol{x}^{\prime}, t^{\prime}\right)\right\rangle=\frac{k_{B} T}{(2 \pi)^{d / 2}}\left|\boldsymbol{x}-\boldsymbol{x}^{\prime}\right|^{z-d} \times \\
& \left\{f\left[\frac{t}{\tau\left(\left|\boldsymbol{x}-\boldsymbol{x}^{\prime}\right|\right)}\right]+f\left[\frac{t^{\prime}}{\tau\left(\left|\boldsymbol{x}-\boldsymbol{x}^{\prime}\right|\right)}\right]-f\left[\frac{\left|t-t^{\prime}\right|}{\tau\left(\left|\boldsymbol{x}-\boldsymbol{x}^{\prime}\right|\right)}\right]\right\},
\end{aligned}
$$

where $\delta_{t} h(\boldsymbol{x}, t)=h(\boldsymbol{x}, t)-h(\boldsymbol{x}, 0)$ and $f[u]$ has been introduced in (4.14) [51]. From (4.14) and (4.17) it follows that the average drift is given by

$$
\langle h(x, t)\rangle_{F_{0}}=\frac{F_{0}}{2 k_{B} T}\left\langle\delta_{t} h(\boldsymbol{x}, t) \delta_{t} h\left(\boldsymbol{x}^{\star}, t\right)\right\rangle .
$$

The former equality constitutes the most general form of the Kubo fluctuation relation (KFR) and encompasses both tagged and untagged particles. Another formulation of the KFR is furnished as follows. Comparing the expression for the untagged probe drift (4.7) with the corresponding expression of the position-velocity correlation function (2.29), we recover the generalized KFR such as $[45,59,60]$

$$
\langle h(\boldsymbol{x}, t)\rangle_{F_{0}}=\frac{F_{0}}{k_{B} T} \int_{0}^{t} d t^{\prime}\left\langle h(\boldsymbol{x}, t) v\left(\boldsymbol{x}^{\star}, 0\right)\right\rangle
$$

which corresponds to (4.18) after integration. In case of the tagged probe we get the (usual) Einstein relation [45]

$$
\left\langle h\left(\boldsymbol{x}^{\star}, t\right)\right\rangle_{F_{0}}=\frac{\left\langle\delta_{t}^{2} h\left(\boldsymbol{x}^{\star}, t\right)\right\rangle}{2 k_{B} T} F_{0}
$$

which corresponds to the limit $\boldsymbol{x} \rightarrow \boldsymbol{x}^{\star}$ of (4.18). One can see that the Einstein relation (4.20) is fulfilled also by untagged probe, provided that $t \gg \tau$, i.e. by the time that the correlation length $\xi(t)=(A t)^{2 / \gamma}$ exceeds the distance $\left|\boldsymbol{x}-\boldsymbol{x}^{\star}\right|$.

To sum up, Eq.(4.18) constitutes the Kubo fluctuation relation for the linear system (1.1) in the case of a local constant force applied in $\boldsymbol{x}^{\star}$. The FLE (2.1) can thus be considered as a stochastic representation of the the KFR (4.18) with the random force $\zeta(\boldsymbol{x}, t)$ satisfying the (second) FD relation $(2.9)$.

\subsection{Edward-Wilkinson chain}

We now test some of the previous results in case of a system with local hydrodynamic interactions and $z=2, d=1$. In this special case Eq.(1.5) reads

$$
\frac{\partial}{\partial t} h(x, t)=A \frac{\partial^{2}}{\partial x^{2}} h(\boldsymbol{x}, t)+F_{0} \theta(t) \delta\left(x^{\star}-x\right)+\eta(\boldsymbol{x}, t),
$$

where $\beta=1 / 2, \gamma=4, A=$ const $>0$ and $K^{+}=2 / \sqrt{A}$ from (2.2), (2.3) and (2.4), respectively. This is, for instance, the equation for the 1-dimensional Rouse polymer [9] or the Edward-Wilkinson chain [7] and it constitutes a benchmark for our analysis since the drift can be calculated directly from Eq.(4.21) in terms of simpler functions.

We start by considering the tagged probe, Eq.(4.9) yields

$$
\left\langle h\left(\boldsymbol{x}^{\star}, t\right)\right\rangle_{F_{0}}=F_{0} \sqrt{\frac{A t}{\pi}} .
$$

For the untagged probe, we make use of Eq.(4.7) plugging in the values of parameters specified above. By using the reduction formula (A.5), the formulae (A.4) and (A.12), we obtain 




After the integration in time we get the final expression

$$
\langle h(\boldsymbol{x}, t)\rangle_{F_{0}}=F_{0}\left[\sqrt{\frac{A t}{\pi}} e^{-\frac{\left|\boldsymbol{x}-\boldsymbol{x}^{\star}\right|^{2}}{4 A t}}-\frac{\left|\boldsymbol{x}-\boldsymbol{x}^{\star}\right|}{2} \operatorname{erfc}\left(\frac{\left|\boldsymbol{x}-\boldsymbol{x}^{\star}\right|}{2 \sqrt{A t}}\right)\right],
$$

where $\operatorname{erfc}$ represents the complementary error function. The same result can be obtained directly from Eq.(4.21) without using Fox $H$-function formalism. We can then study short and long time limits of the former equation. For $t \ll \frac{\left(\boldsymbol{x}-\boldsymbol{x}^{\star}\right)^{2}}{A}$ we have

$$
\langle h(\boldsymbol{x}, t)\rangle_{F_{0}} \simeq \frac{2}{\sqrt{\pi}\left|\boldsymbol{x}-\boldsymbol{x}^{\star}\right|^{2}} F_{0}(A t)^{3 / 2} e^{-\frac{\left|\boldsymbol{x}-\boldsymbol{x}^{\star}\right|^{2}}{4 A t}},
$$

which matches the expression (4.13). For $t \gg \frac{\left|\boldsymbol{x}-\boldsymbol{x}^{\star}\right|^{2}}{A}$ instead the asymptotic drift is given by Eq.(4.22).

\section{Conclusions}

In this paper we derived the FLE for tagged and untagged probe particle in a generalized elastic model where a localized force is supposed to operate. The tagged probe is considered to be the point on which the external force acts, while the untagged probe is any other point on the system, which is secondarily affected by the action of the perturbation. Within the FLE framework, the stochastic motion of both tracers evidently appears to be influenced by the external force, since the external perturbation propagates through the system. This propagation is mathematically expressed by the noise and force propagators (Green's functions), carrying the perturbation between two points of the system, in a certain lapse of time. We analyzed the double scaling behaviour of these propagators which arise naturally thanks to Fox $H$-function formalism. We have shown that such behaviour affects the stochastic dynamics of both tracers. In particular, we demonstrated how the response of the untagged tracer differs drastically for long and short time, in the case of a constant force applied, when hydrodynamic interactions can be considered long-ranged or local.

We related our general theoretical set-up to the specific case of Edward-Wilkinson chain with a local constant force. Importantly, we have shown how the Fox $H$-function formalism constitutes a powerful, compact and elegant way to express the observables and allows the straightforward study of their asymptotic behaviours.

Appendix

\section{A. Fox function properties}

In this Section we enumerate the properties of the Fox $H$-functions that we use throughout our analysis. This list is not an exhaustive compendium of the Fox $H$-function properties, for which the reader could refer to $[33,34,44]$. Some useful properties are also reported in [31].

Fox $H$-functions are defined through the Mellin transform

$$
H_{p q}^{m n}\left[y \mid \begin{array}{ccc}
\left(a_{1}, A_{1}\right) & \ldots & \left(a_{p}, A_{p}\right) \\
\left(b_{1}, B_{1}\right) & \ldots & \left(b_{q}, B_{q}\right)
\end{array}\right]=\frac{1}{2 \pi i} \int_{L} \chi(s) y^{-s} d s
$$

with $1 \leq m \leq q, 0 \leq n \leq p$. $\chi(s)$ is given by

$$
\chi(s)=\frac{\prod_{j=1}^{m} \Gamma\left(b_{j}+B_{j} s\right) \prod_{j=1}^{n} \Gamma\left(1-a_{j}-A_{j} s\right)}{\prod_{j=m+1}^{q} \Gamma\left(1-b_{j}-B_{j} s\right) \prod_{j=n+1}^{p} \Gamma\left(a_{j}+A_{j} s\right)} .
$$


where $A_{j}$ and $B_{j}$ are positive numbers while $a_{j}$ and $b_{j}$ are complex. Empty products are interpreted as being unity.

For convenience in this Section we adopt the following short notation

$$
H_{p q}^{m n}\left[y \mid \begin{array}{ll}
\left(a_{1}, A_{1}\right) \ldots\left(a_{p}, A_{p}\right) \\
\left(b_{1}, B_{1}\right) \ldots & \left(b_{q}, B_{q}\right)
\end{array}\right]=H_{p q}^{m n}\left[y \mid \begin{array}{c}
{\left[a_{p}, A_{p}\right]} \\
{\left[b_{q}, B_{q}\right]}
\end{array}\right] .
$$

The useful rules are hereafter listed:

$$
\begin{aligned}
& H_{p q}^{m n}\left[y \mid \begin{array}{l|l}
{\left[a_{p}, A_{p}\right]} \\
{\left[b_{q}, B_{q}\right]}
\end{array}\right]=H_{q p}^{n m}\left[\begin{array}{l|l}
\frac{1}{y} & {\left[1-b_{q}, B_{q}\right]} \\
{\left[1-a_{p}, A_{p}\right]}
\end{array}\right], \\
& H_{p q}^{m n}\left[y \mid \begin{array}{c}
{\left[a_{p}, A_{p}\right]} \\
{\left[b_{q}, B_{q}\right]\left(a_{1}, A_{1}\right)}
\end{array}\right]=H_{p-1 q-1}^{m n-1}\left[y \mid \begin{array}{c}
\left(a_{2}, A_{2}\right), \ldots .,\left(a_{p}, A_{p}\right) \\
{\left[b_{q-1}, B_{q-1}\right]}
\end{array}\right], \\
& \int_{0}^{\infty} d y y^{\alpha-1} \cos (\sigma y) H_{p q}^{m n}\left[\omega y^{r} \mid \begin{array}{l|l}
{\left[a_{p}, A_{p}\right]} \\
{\left[b_{q}, B_{q}\right]}
\end{array}\right]=
\end{aligned}
$$

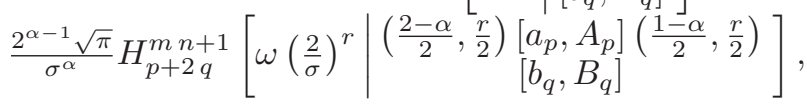

$$
\begin{aligned}
& \int_{0}^{x} d y y^{\alpha-1} H_{p q}^{m n}\left[\omega y \mid \begin{array}{l}
{\left[a_{p}, A_{p}\right]} \\
{\left[b_{q}, B_{q}\right]}
\end{array}\right]= \\
& x^{\alpha} H_{p+1 q+1}^{m n+1}\left[\omega x \mid \begin{array}{c|c}
(1-\alpha, 1)\left[a_{p}, A_{p}\right] \\
{\left[b_{q}, B_{q}\right](-\alpha, 1)}
\end{array}\right], \\
& \frac{1}{k} H_{p q}^{m n}\left[y \mid \begin{array}{l}
{\left[a_{p}, A_{p}\right]} \\
{\left[b_{q}, B_{q}\right]}
\end{array}\right]=H_{p q}^{m n}\left[\begin{array}{l|l}
k & {\left[\begin{array}{l}
\left.a_{p}, k A_{p}\right] \\
{\left[b_{q}, k B_{q}\right]}
\end{array}\right]}
\end{array}\right], \\
& y^{\sigma} H_{p q}^{m n}\left[y \mid \begin{array}{l}
{\left[a_{p}, A_{p}\right]} \\
{\left[b_{q}, B_{q}\right]}
\end{array}\right]=H_{p q}^{m n}\left[y \mid \begin{array}{l}
{\left[a_{p}+\sigma A_{p}, A_{p}\right]} \\
{\left[b_{q}+\sigma B_{q}, B_{q}\right]}
\end{array}\right], \\
& \int_{0}^{\infty} d y y^{\alpha-1} J_{\nu}(\sigma y) H_{p q}^{m n}\left[\omega y^{r} \mid \begin{array}{l}
{\left[a_{p}, A_{p}\right]} \\
{\left[b_{q}, B_{q}\right]}
\end{array}\right]=
\end{aligned}
$$

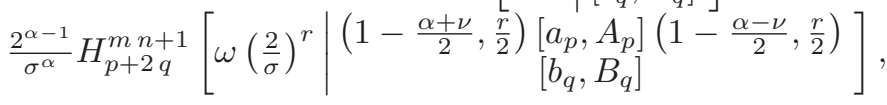



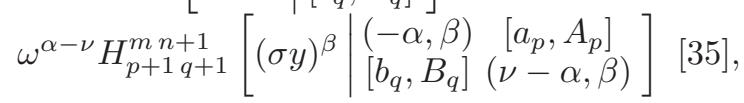

$$
\begin{aligned}
& H_{01}^{10}\left[y \mid \begin{array}{c}
- \\
{\left[b_{q}, B_{q}\right]}
\end{array}\right]=\frac{y^{\frac{b}{B}}}{B} e^{-y^{1 / B}} .
\end{aligned}
$$

Asymptotic expansion: $y \rightarrow 0$,

$$
\begin{aligned}
& H_{p q}^{m n}\left[y \mid \begin{array}{l}
{\left[a_{p}, A_{p}\right]} \\
{\left[b_{q}, B_{q}\right]}
\end{array}\right]=\sum_{i=1}^{m} \sum_{k=0}^{\infty} c_{i k} \frac{(-1)^{k}}{k ! B_{i}} y^{\frac{b_{i}+k}{B_{i}}} \\
& c_{i k}=\frac{\prod_{j=1, j \neq i}^{m} \Gamma\left(b_{j}-\frac{\left(b_{i}+k\right) B_{j}}{B_{i}}\right) \prod_{j=1}^{n} \Gamma\left(1-a_{j}+\frac{\left(b_{i}+k\right) A_{j}}{B_{i}}\right)}{\prod_{j=m+1}^{q} \Gamma\left(1-b_{j}+\frac{\left(b_{i}+k\right) B_{j}}{B_{i}}\right) \prod_{j=n+1}^{p} \Gamma\left(a_{j}-\frac{\left(b_{i}+k\right) A_{j}}{B_{i}}\right)} .
\end{aligned}
$$

This expansion is valid whenever $\sum_{i=1}^{q} B_{i}-\sum_{i=1}^{p} A_{i} \geq 0$ or $\sum_{i=1}^{q} B_{i}-\sum_{i=1}^{p} A_{i}<0$ and $\sum_{i=1}^{n} A_{i}-$ $\sum_{i=n+1}^{p} A_{i}+\sum_{i=1}^{m} B_{i}-\sum_{i=m+1}^{q} B_{i}>0$.

Asymptotic expansion: $y \rightarrow \infty$, 


$$
\begin{aligned}
& H_{p q}^{m n}\left[y \mid \begin{array}{l}
{\left[a_{p}, A_{p}\right]} \\
{\left[b_{q}, B_{q}\right]}
\end{array}\right]=\sum_{i=1}^{n} \sum_{k=0}^{\infty} c_{i k} \frac{(-1)^{k}}{k ! A_{i}} y \frac{-\left(1-a_{i}+k\right)}{A_{i}} \\
& c_{i k}=\frac{\prod_{j=1, j \neq i}^{n} \Gamma\left(1-a_{j}-\frac{\left(1-a_{i}+k\right) A_{j}}{A_{i}}\right) \prod_{j=1}^{m} \Gamma\left(b_{j}+\frac{\left(1-a_{i}+k\right) B_{j}}{A_{i}}\right)}{\prod_{j=n+1}^{p} \Gamma\left(a_{j}+\frac{\left(1-a_{i}+k\right) A_{j}}{A_{i}}\right) \prod_{j=m+1}^{q} \Gamma\left(1-b_{j}-\frac{\left(1-a_{i}+k\right) B_{j}}{A_{i}}\right)} .
\end{aligned}
$$

This expansion is valid whenever $\sum_{i=1}^{q} B_{i}-\sum_{i=1}^{p} A_{i} \leq 0$ or $\sum_{i=1}^{q} B_{i}-\sum_{i=1}^{p} A_{i}>0$ and $\sum_{i=1}^{n} A_{i}-$ $\sum_{i=n+1}^{p} A_{i}+\sum_{i=1}^{m} B_{i}-\sum_{i=m+1}^{q} B_{i}>0$. Empty products are interpreted as being unity.

Acknowledgements. A.T. acknowledges the financial support of the European Complexity-net pilot project "LOCAT".

\section{References}

[1] A. Taloni, A. Chechkin, J. Klafter. Generalized elastic model yields a fractional Langevin equation description. Phys. Rev. Lett. 104 (2010), No 16, 160602-1-4.

[2] A. Saichev, M. Zazlawsky. Fractional kinetic equations: solutions and applications. Chaos, 7 (1997), No 4, $753-765$.

[3] S. G. Samko, A. A. Kilbas, O. I. Marichev. Fractional Integrals and Derivatives, Theory and Applications. Gordon and Breach, Amsterdam, 1993.

[4] M. Doi, S. F. Edwards. The Theory of Polymer Dynamics. Clarendon, Oxford, 1986.

[5] R. Granek. From semi-flexible polymers to membranes: anomalous diffusion and reptation. J. Phys. II France, 7 (1997), 1761-1788.

[6] E. Farge, A. C. Maggs. Dynamic scattering from semiflexible polymers. Macromol., 26 (1993), No 19, 5041-5044. A. Caspi, M. Elbaum, R. Granek, A. Lachish, D. Zbaida. Semiflexible polymer network: a view from inside. Phys. Rev. Lett., 80 (1998), No 5, 1106-1109. F. Amblard, A. C. Maggs, B. Yurke, A. N. Pargellis, S. Liebler Subdiffusion and anomalous local viscoelasticity in acting networks. Phys. Rev. Lett., 77 (1996), No 21, 4470-4473.

[7] S. F. Edwards, D. R. Wilkinson. The surface statistics of a granular aggregate. Proc. R. Soc. London A, 381 (1982), No $1780,17-31$.

[8] B. H. Zimm. Dynamics of polymer molecules in dilute solution: viscoelasticity flow birefringence and dielectric loss. J. Chem. Phys., 24 (1956), No 2 269-278 .

[9] P. E. Rouse. A theory of the linear viscoelastic properties of dilute solutions of coiling polymers. J. Chem. Phys., 21 (1953), No 7, 1272-1280.

[10] E. Freyssingeas, D. Roux, F. Nallet. Quasi-Elastic Light Scattering Study of Highly Swollen Lamellar and "Sponge" Phases. J. Phys. II France, 7 (1997), 913-929. E. Helfer, S. Harlepp, L. Bourdieu, J. Robert, F. C. MacKintosh, D. Chatenay. Microrheology of biopolymer-membrane complexes. Phys. Rev. Lett., 85 (2000), No 2, 457-460.

[11] R. Granek, J. Klafter. Anomalous motion of membranes under a localized external potential. Europhys. Lett., 56 (2001), No 1, 15-21.

[12] A. G. Zilman, R. Granek. Membrane dynamics and structure factor. Chem. Phys., 284 (2002), 195-204.

[13] A. G. Zilman, R. Granek. Dynamics of fractal sol-gel polymeric clusters. Phys. Rev. E, 58 (1998), No 3, R2725-R2728.

[14] P. C. Searson, R. Li, K. Sieradzki. Surface Diffusion in the Solid-on-Solid Model. Phys. Rev. Lett. 74 (1995), No 8, 1395-1398. J. Krug, H. T. Dobb. Anomalous Tracer Diffusion on Surfaces. Phys. Rev. Lett. 76 (1996), No 21, 4096-4096. S. N. Majumdar, A. Bray. Spatial persistence of fluctuating interfaces. Phys. Rev. Lett. 86 (2001), No 17, 3700-3703. For a review on fluctuating interfaces, see J. Krug. Origins of scale invariance in growth processes . Adv. Phys., 46 (1997), No 2, 139-282 and J. Krug in Scale Invariance, Interfaces and Non-Equilibrium Dynamics. edited by A. McKane et al. Plenum, New York, 1995.

[15] J. Krug, H. Kallabis, S. N. Majumdar, S. J. Cornell, A. J. Bray, C. Sire. Persistence exponents for fluctuating interfaces. Phys. Rev. E, 56 (1997), No 3, 2702-2712.

[16] Z. Toroczkai, E. D. Williams. Nanoscale fluctations at solid surfaces. Phys. Today, 52 (1998), No.12 , 24-29.

[17] S. Majaniemi, T. Ala-Nissila, J. Krug. Kinetic roughening of surfaces: Derivation, solution, and application of linear growth equations. Phys. Rev. B, 53 (1995), No 12, 8071-8082.

[18] H. Gao, J. R. Rice. A first order perturbation analysis of crack trapping by arrays of obstacles. J. Appl. Mech., 65 (1989), No 56, 828-836.

[19] J. F. Joanny, P. G. de Gennes. A model for contact angle hysteresis. J. Chem. Phys., 81 (1984), 552-549.

[20] F. Mainardi, P. Pironi. The Fractional Langevin Equation: Brownian Motion Revisited. Extr. Math. 10 (1996), No 1, 140-154; F. Mainardi, A. Mura and F. Tampieri. Brownian motion and anomalous diffusion revisited via a fractional Langevin equation. Modern Problems of Statistical Physics 8 (2009), 3-23.

[21] E. Lutz. Fractional Langevin equation. Phys. Rev. E, 64 (2001), No 5, 051106-1-4. E. Lutz. Fractional Langevin Eqaution. In Fractional Dynamics. Recent Advances. edited by J. Klafter, S. C. Lim and R. Metzler. World Scientific, Singapore, 2012. Ch.12, pp. 285-305.

[22] B. B. Mandelbrot, J. W. Van Ness, Fractional Brownian Motions, Fractional Noises and Applications. SIAM Rev., 10 (1968), 422-437. 
[23] S. C. Kou, X. S. Xie. Generalized Langevin equation with fractional Gaussian noise: subdiffusion within a single protein molecule. Phys. Rev. Lett., 93 (2004), No 18, 180603-1-4. S. C. Kou. Stochastic Modeling in Nanoscale Biophysics: Subdiffusion within Proteins. Annals Applied Statistics, 2 (2008), No 2, 501-535.

[24] S. Burov, E. Barkai. Critical exponent of the fractional Langevin equation. Phys. Rev. Lett., 100 (2008), No 7, 070601$1-4$.

[25] K. Sau Fa. Generalized Langevin equation with fractional derivative and long-time correlation function. Phys. Rev. E, 73 (2006), No 6, 061104-1-4.

[26] K. Sau Fa. Fractional Langevin equation and Riemann-Liouville fractional derivative. Eur. Phys. J. E, 24 (2007), No 2, 139-143.

[27] I. Goychuk. Viscoelastic Subdiffusion: Generalized Langevin Equation Approach. Adv. Chem. Phys., 150 (2012), 187253.

[28] A. Taloni, M. A. Lomholt. Langevin formulation for single-file diffusion. Phys. Rev. E, 78 (2008), No 5, 051116-1-8.

[29] L. Lizana, T. Ambjornsson, A. Taloni, E. Barkai, M A. Lomholt. et al.. Foundation of fractional Langevin equation: Harmonization of a many-body problem. Phys. Rev. E, 81 (2010), No 5, 051118-1-8.

[30] D. Panja. Generalized Langevin equation formulation for anomalous polymer dynamics. J. Stat. Mech., (2010), L020011-8. D. Panja. Anomalous polymer dynamics is non-Markovian: memory effects and the generalized Langevin equation formulation. J. Stat. Mech., (2010), P06011-1-34.

[31] A. Taloni, A. Chechkin, J. Klafter. Correlations in a generalized elastic model: Fractional Langevin equation approach. Phys. Rev. E, 82 (2010), No 6, 061104-1-15.

[32] C. Fox. The $G$ and $H$ Functions as symmetrical Fourier kernels. Trans. Amer. Math. Soc., 98 (1961), $395-429$.

[33] A. M. Mathai, R. K. Saxena. The H-Function with Application in Statistics and Other Discplines. Wiley Eastern Limited, New Delhi-Bangalore-Bombay, 1978.

[34] R. Hilfer (Ed.). Applications of Fractional Calculus in Physics. World Scientific, Singapore, 2000.

[35] W. G. Gloeckle, T. F. Nonnenmacher. Fractional Integral Operators and Fox Functions in the Theory of Viscoelasticity. Macromolecules, 24 (1991), 6426-6434. G. Gloeckle, T. F. Nonnenmacher. Fox Function Representation of Non-Debye Relaxation Processes. Journ. Stat. Phys. 71 (1993), Nos 3/4, 741-757.

[36] R. Metzler, W. G. Gloeckle, T. F. Nonnenmacher, Fractional model equation for anomalous diffusion. Physica A, 211 (1994), 13-24. R. Metzler, J. Klafter. The Random Walk Guide to Anomalous Difffusion: a Fractional Dynamics Approach. Phys. Rep. 339 (2000), 1-77.

[37] F. Mainardi, G. Pagnini, R. K. Saxena. Fox H functions in fractional diffusion. Journ. Comput. Applied Math., 178 (2005), Nos 1-2, 321-331.

[38] S. I. Denisov, S. B. Yuste, Yu. S. Bystrik, H. Kantz, K. Lindenberg. Asymptotic solutions of decoupled continuous-time random walks with superheavy-tailed waiting time and heavy-tailed jump length distributions. Phys. Rev. E, 84 (2011), 061143-1-7.

[39] R. K. Saxena, A. M. Mathai, H. J. Haubold. Fractional Reaction-Diffusion Equations. Astrophys Space Sci, 305 (2006), 289-296.

[40] M. O. Vlad, R. Metzler, J. Ross. Generalized Huber kinetics for nonlinear rate processes in disordered systems: Nonlinear analogs of stretched exponential. Phys. Rev. E, 57 (1998), No. 6, 6497-6505.

[41] N. Laskin. Fractional quantum mechanics and Lev́y path integrals. Phys. Lett. A, 268 (2000), 298-305. N. Laskin. Fractals and quantum mechanics. Chaos, 10 (2000), No. 4, 780-790. N. Laskin. Fracional quantum mechanics. Phys. Rev. E 62, (2000), No.3, 3135-3145. N. Laskin. Lev́y flights over quantum paths. Communications Nonlinear Sci and Numer. Simulations, 12 (2007), No. 1, 2-18. J. Dong. Green's function for the time-dependent scattering problem in the fractional quantum mechanics. Journ. Math. Phys., 52 (2011), 042103-1-10.

[42] A. A. Kilbas, M. Saigo, H-Transforms. Theory and Applications. Chapman and Hall, London, 2004.

[43] A. M. Mathai, R. K. Saxena, H. J. Haubold, The H - Function. Theory and Applications. Springer, New York, 2010.

[44] A. P. Prudnikov, Y. A. Brychkov, O. I. Marichev. Integral and Series. Vol.3: More special Functions. Gordon and Breach Science, Amsterdam, 1990.

[45] A. Taloni, A. Chechkin, J. Klafter. Unusual response to a localized perturbation in a generalized elastic model. Phys. Rev. E, 84 (2011), No 2, 021101-1-7.

[46] E. Helfer, S. Harlepp, L. Bourdieu, J. Robert, F. C. MacKintosh, D. Chatenay. Microrheology of biopolymer-membrane complexes. Phys. Rev. Lett., 85 (2000), No 2 457-460. E. Helfer, S. Harlepp, L. Bourdieu, J. Robert, F. C. MacKintosh, D. Chatenay. Viscoelastic properties of actin-coated membranes. Phys. Rev. E, 63 (2001), No 2, 021904-1-13.

[47] Chau-Hwang Lee et al. Three-Dimensional Characterization of Active Membrane Waves on Living Cells. Phys. Rev. Lett. 103 (2009), No 23, 238101-1-4.

[48] I. Podlubny. Fractional Differential Equations. Academic Press, New York, 1999.

[49] M. Caputo. Linear model of dissipation whose $Q$ is almost frequency independent. Geophys. J. R. Astr. Soc., 13 (1967), 529-539.

[50] D. C. Champeney. Fourier Transforms and Physical Applications. Academic Press, London, 1973.

[51] A. Taloni, A. Chechkin, J. Klafter. Generalized elastic model: Thermal vs. non-thermal initial conditions. Universal scaling, roughening, ageing and ergodicity. Europhys. Lett., 97 (2012), No 3, 30001-p1-p6.

[52] A. L. Barabasi, H. E. Stanley. Fractal Concepts in Surface Growth. Cambridge University Press, Cambridge, 1994

[53] P. Meakin. Fractal and scaling growth far from equilibrium. Cambridge University Press, Cambridge, 1998.

[54] F. Family, T. Vicsek. Scaling of the active zone in the Eden process on percolation networks and the ballistic deposition model. J. Phys. A: Math. Gen., 18 (1985), No 2, L75-L81. 
[55] M. Abramowitz, I. Stegun. Handbook of Mathematical Functions. Dover, New York, 1964.

[56] A. P. Prudnikov, Y. A. Brychkov, O. I. Marichev. Integrals and Series. Vol.I: Elementary Functions. New York: Gordon and Breach, 1986.

[57] I. M. Gelfand, G. E. Shilov, Generalized Functions. Academic Press, 1964.

[58] A. W. J. Erdelyi. Asymptotic Expansions. Dover, New York, 1956.

[59] R. Kubo, M. Toda, N. Hatsushime. Statistical Physics II: Nonequilibrium Statistical Mechanics. Springer, Berlin, 1991.

[60] U. M. B. Marconi, A. Puglisi, L. Rondoni, A. Vulpiani. Fluctuation-dissipation: response theory in statistical physics. Phys. Rep., 461 (2008), No 4-6, 111-195. D. Villamaina, A. Baldassarri1, A. Puglisi, A. Vulpiani. The fluctuationdissipation relation: how does one compare correlation functions and responses? . J. Stat. Mech., (2009), P07024-1-22.

[61] E. Barkai, R. Silbey. Theory of Single File Diffusion in a Force Field. Phys. Rev. Lett., 102 (2009), No 5, 050602-1-4.

[62] I. S. Gradshtein, I. M. Rizhikl. Tables of Integrals. Series and Products. Academic Press, New York, 2007.

[63] R. Santachiara, A Rosso, W Krauth. Universal width distributions in non-Markovian Gaussian processes. J. Stat. Mech., (2007), P02009-.

[64] G. H. Hardy. Divergent Series. Clarendon Press, Oxford, 1949.

[65] F. W. J. Olver. Asymptotics and Special Functions. Academic Press, New York, 1974. 